\title{
Consensus Control Laws with Guaranteed Performance in Presence of Fixed Delays
}

\author{
Prathyush P Menon, Alexandre Seuret, and Christopher Edwards
}

\begin{abstract}
This paper deals with the design of optimal consensus control laws for a set of identical first and second order agents. Delayed versions of the relative states with respect to neighbours is used in the proposed consensus laws. Two different approaches are adopted: (i) assuming no delay is present in the exchange of information, design an optimal consensus law and for those gains, determine the maximum bound on the acceptable delay before stability deteriorates (ii) develop optimal consensus laws using a novel algorithm which explicitly accounts for a known fixed delay at the time of design itself. Optimal designs for a network of first and second order agents obtained using the two different approaches.
\end{abstract}

\section{INTRODUCTION}

Recently, there has been significant research effort to analyze the impact of different types of delays on the stability and performance of network level dynamics - references [1][12] are few examples, however the list is not exhaustive. ${ }^{1}$ Necessary and sufficient conditions for average consensus problems in networks of linear agents in the presence of communication delays have been derived in [1], also see [2] for the development of sufficiency conditions for the existence of average consensus in the presence of bounded delay, both constant and time varying, from the perspective of a time-delay partial difference equation. Stability criteria for the consensus dynamics in networks of agents in the presence of communication delays was subsequently developed in [3] using Lyapunov Krasovskii techniques and the strong dependence of the magnitude of delay and the initial conditions on the consensus value was also established.

In [4], a network of second order dynamical systems with heterogeneously delayed exchange of information between agents is considered, and flocking or rendezvous is obtained using decentralized control. This can be tuned locally, based only on the delays associated with the local neighbours. Both frequency and time domain approaches are utilized in [4] to establish delay dependent and independent collective stability. Subsequently the theory in [4] was extended in [6]

Prathyush P. Menon and Christopher Edwards are affiliated to the Center for Systems, Dynamics and Control, College of Engineering, Mathematics, and Physical Sciences, University of Exeter, Exeter EX4 4QF, UK P.M.Prathyush@exeter.ac.uk, C. Edwards dexeter.ac.uk Alexandre Seuret is with the Methods and Algorithms in Control (MAC) team at LAAS-CNRS, Toulouse, France. alexandre.seuretelaas.fr

This work has been funded by the ANR under Grant LIMI- COS ANR12-BS03-005 and the UKIERI grant IND/COM/E/11- 12/097.

${ }^{1}$ Another research area involving the stabilization of time-delay systems is networked control systems [13], [14]. This is not the class of problems considered in this paper. to the case of a network formed from a certain class of nonlinear systems. The robustness of linear consensus algorithms and conditions for convergence subject to node level self delays and relative measurement delays were developed and reported in [5] building on the research described in [4] and [6]. 'Scalable' delay dependent synthesis of consensus controllers for linear multi agent networks making use of delay dependent conditions is proposed in [5].

Reference [7] reports an independent attempt to achieve second order consensus using delayed position and velocity information. Recently another methodology, based on a cluster treatment of characteristic roots, has been proposed in [8] to study the effect of large and uniform delays in second order consensus problems with undirected graphs. In [9] the performance of consensus algorithms in terms of providing a fast convergence rate involving communication delays, was studied for second order multi agent systems. In [10] consensus problem was studied based on using position information and delayed relative position information only.

In this paper, two distinct approaches are proposed to synthesize consensus laws in linear multi agent systems ensuring a certain level of performance in the presence of homogenous self delays. In the first approach, the delay is first ignored, and consensus laws are developed minimizing a performance cost associated with consensus. Subsequently, the maximum acceptable delay that could be accommodated is assessed, not violating the stability condition, using the traditional Nyquist theory. In the second approach, it is proposed to explicitly account the delay in communication while designing the consensus law that ensure a level of LQR-like performance.

\section{A. Notations and Preliminaries}

Standard notations are employed in the paper. The set of real numbers is denoted by $\mathbb{R}$, and $\mathbb{R}^{n}$ and $\mathbb{R}^{n \times n}$ represent the set of $n$ dimensional vectors, and the set of $n \times m$ matrices, where $n$ and $m$ are positive integers. The set of symmetric positive definite matrices are represented by $\mathbb{S}_{n}^{+}$. For all $M \in \mathbb{R}^{n \times n}$, the notation $\mathcal{H} e\{M\}$ stands for $M+M^{T}$. The representations $\mathcal{C}$ ol(.) and $\mathcal{D} \operatorname{iag}($.$) denote a column$ vector and diagonal matrix respectively. For $x \in \mathbb{R}^{n},|x|$ is the euclidian norm of the vector $x$. An $n \times n$ identity matrix is denoted by $I_{n}$. The Kronecker product is denoted by the symbol $\otimes$.

The notations for time delay systems are standard. For $\tau>$ 0 , the notation $x_{t}$ refers to a function defined over the time interval $[-\tau, 0]$ such that for all $\theta \in[-\tau, 0], x_{t}(\theta)=x(t+$ $\theta)$. Additionally, the norm $\left\|x_{t}\right\|_{\tau}=\sup _{\theta \in[-\tau, 0]}|x(t+\theta)|$. 
Details on graph theory can be found in standard texts such as [15]. However, the relevant basic concepts used in this paper are described in this section. Bidirectional communication among agents is assumed, and these networks are represented in terms of undirected graphs. The adjacency matrix for the graph $\mathcal{A}(\mathcal{G})=\left[a_{i j}\right]$, is defined by $a_{i j}=1$ if $i$ and $j$ are adjacent nodes of the graph, and $a_{i j}=0$ otherwise. The degree matrix is represented by the symbol $\Delta(\mathcal{G})=\left[\delta_{i j}\right] . \Delta(\mathcal{G})$ is a diagonal matrix, and each element $\delta_{i i}$ is the degree of the $i^{t h}$ vertex. The difference $\Delta(\mathcal{G})-\mathcal{A}(\mathcal{G})$ defines the Laplacian of $\mathcal{G}$, written as $\mathcal{L}$. For an undirected graph, $\mathcal{L}$ is symmetric positive semi-definite. The smallest eigenvalue of the Laplacian $\mathcal{L}$ is zero and the corresponding eigenvector is given by $\mathbf{1}=\operatorname{Col}(1, \ldots 1)$. The Laplacian is always rank deficient and the rank of $\mathcal{L}$ is $n-1$ if and only if $\mathcal{G}$ contains a spanning tree. The maximal eigenvalue of the $\mathcal{L}$ is bounded by $2 \max _{i} \delta_{i i}$ [16].

\section{Problem Formulation}

\section{A. System description}

Consider a network of $N$ identical single/double integrator agents represented as

$$
\dot{x}_{i}(t)=A x_{i}(t)+B u_{i}(t)
$$

for $i=1, \ldots, N$, where $x_{i}(t) \in \mathbb{R}^{n}$ and $u_{i}(t) \in \mathbb{R}$ are the states and control inputs respectively. Each agent (node) is assumed to share relative state information with its neighbours, according to

$$
z_{i}(t)=\sum_{j \in \mathcal{N}_{i}}\left(x_{i}(t-\tau)-x_{j}(t-\tau)\right) .
$$

In (2), the scalar self delay term $\tau$ is assumed to be known, fixed, and identically present in the communication of the relative information. The set $\mathcal{N}_{i} \subset\{1,2, \ldots, N\} /\{i\}$ is the neighbourhood set of the $i^{t h}$ agent, and consists of all those agents with which the $i^{\text {th }}$ agent can communicate.

\section{B. Design Objectives}

The intention is to design control laws of the form

$$
u_{i}(t)=-K z_{i}(t)
$$

to obtain consensus such that the performance cost

$$
J_{i}:=\int_{0}^{\infty} \sum_{j \in \mathcal{N}_{i}}\left(\left(x_{i}(t)-x_{j}(t)\right)^{T} Q\left(x_{i}(t)-x_{j}(t)\right)\right)+u_{i}(t)^{T} R u_{i}(t) d t,
$$

is minimized for all $i=1, \ldots, N$, where the matrix $K \in$ $\mathbb{R}^{1 \times n}$ represents the gain and $Q \in \mathbb{R}^{n \times n}$ and $R \in \mathbb{R}^{m \times m}$ are pre-specified symmetric positive definite matrices. The first term of the performance cost function (4) (different to a conventional LQR cost) is introduced to ensure consensus performance. The second term in (4) is a standard term associated with the control effort.

\section{DESIGN APPROACH}

\section{A. Network representation}

Using a Kronecker product representation, the system in (1) at network level is

$$
\dot{X}(t)=\left(I_{N} \otimes A\right) X(t)+\left(I_{N} \otimes B\right) U(t),
$$

where the augmented state $X(t)=\mathcal{C} o l\left(x_{1}(t), \ldots, x_{N}(t)\right)$ and $U(t)=\mathcal{C}$ ol $\left(u_{1}(t), \ldots, u_{N}(t)\right)$. The control law can be written as

$$
U(t)=-(\mathcal{L} \otimes K) X(t-\tau)
$$

where $\mathcal{L}$ is the Laplacian matrix representing the topology of the network characterized by the sets $\mathcal{N}_{i}$. Substituting (6) into (5), the closed loop system is

$$
\dot{X}(t)=\left(I_{N} \otimes A\right) X(t)-(\mathcal{L} \otimes B K) X(t-\tau) .
$$

\section{B. Model transformation}

Since $\mathcal{L}$ is symmetric positive semi-definite, by spectral decomposition $\mathcal{L}=V \Lambda V^{T}$ where $V \in \mathbb{R}^{N \times N}$ is an orthogonal matrix formed from the eigenvectors of $\mathcal{L}$ and $\Lambda=\operatorname{Diag}\left(\lambda_{1}, \ldots, \lambda_{N}\right)$ is the matrix of eigenvalues of $\mathcal{L}$, all of which are real and ordered such that $\lambda_{1}=0<$ $\lambda_{2} \leq \ldots \leq \lambda_{N}$. As in [10], considering an orthogonal state transformation,

$$
X \rightarrow\left(V^{T} \otimes I_{n}\right) X=: \tilde{X}
$$

the network level closed loop system can be represented in terms of $N$ decoupled systems

$$
\dot{\tilde{x}}_{i}(t)=A \tilde{x}_{i}(t)-\lambda_{i} B K \tilde{x}_{i}(t-\tau),
$$

for $i=1, \ldots, N$, where $\tilde{x}_{i} \in \mathbb{R}^{n}$ represents the states of the $i^{\text {th }}$ agent in the new coordinates.

\section{Performance cost at network level}

At network level, the performance cost can be represented in terms of the augmented state vector $X(t)$ by using (6) for the control vector $U(t)$ as

$$
J=\int_{0}^{\infty} X(t)^{T}\left((\mathcal{L} \otimes Q)+\left(\mathcal{L}^{2} \otimes K^{T} R K\right)\right) X(t) d t .
$$

In the coordinates of (8), and since $V^{T} \mathcal{L}^{2} V=\Lambda^{2}$, the performance cost (10) can be written as

$$
J=\int_{0}^{\infty} \tilde{X}(t)^{T}\left((\Lambda \otimes Q)+\left(\Lambda^{2} \otimes K^{T} R K\right)\right) \tilde{X}(t) d t .
$$

The matrix $\Lambda^{2}$ is diagonal since $\Lambda$ is diagonal, and therefore

$$
J=\sum_{i=2}^{N} \tilde{J}_{i}
$$

where

$$
\tilde{J}_{i}=\int_{0}^{\infty} \tilde{x}_{i}(t)^{T}\left(\lambda_{i} Q+\lambda_{i}^{2} K^{T} R K\right) \tilde{x}_{i}(t) d t,
$$

by noting that $\lambda_{1}=0$. 
For design purpose, the plants in (9) will be considered as belonging to the polytopic system

$$
\tilde{\mathcal{P}}(\rho): \dot{\tilde{x}}_{i}(t)=A \tilde{x}_{i}(t)+\rho \tilde{A}_{i} \tilde{x}_{i}(t-\tau),
$$

where $1 \leq \rho \leq \lambda_{N} / \lambda_{2}$ and $\tilde{A}_{i}=\lambda_{2} B K$. Note that the upper-bound $\lambda_{N} / \lambda_{2}$, is often associated with performance of synchronization[17].

Two distinct approaches are now considered to synthesize control laws subject to the performance cost in (13):

- Approach 1: The delay $\tau$ in (2) is assumed to be zero, and a conventional LQR approach is followed to synthesis the gain $K$ ensuring the performance cost in (10) is minimised. Then based on Nyquist theory, explicit bounds on the maximum delay, $\tau$, for which the control laws ensure stability/performance are determined. The LQR framework automatically provides phase margin guarantees, which are important to this methodology.

- Approach 2: The delay $\tau$ is explicitly accounted for when designing the gains $K$ to ensure a sub optimal level of performance with respect to the (LQR-like) cost in (10). Novel linear matrix inequality conditions are derived.

\section{Design Approach 1}

Under the assumption $\tau=0$, the systems in (9) become

$$
\dot{\tilde{x}}_{i}(t)=A(\rho) \tilde{x}_{i}(t)
$$

where $A(\rho):=A-\rho \lambda_{2} B K$ for $\rho \in\left[1, \lambda_{N} / \lambda_{2}\right]$. Note that in (12) the $x_{1}$ dynamics play no part in the choice of the gain $K$. Choose a quadratic Lyapunov function candidate $V\left(\tilde{x}_{i}(t)\right)=\tilde{x}_{i}(t)^{T} P \tilde{x}_{i}(t)$ where $P \in \mathbb{S}_{n}^{+}$. For the systems in (15), for $i=2, \ldots, N$, consider the matrix inequality

$$
P A(\rho)+A(\rho)^{T} P+\rho \lambda_{2} Q+\rho^{2} \lambda_{2}^{2} K^{T} R K<0 .
$$

If (16) is satisfied then

$$
\dot{V}\left(\tilde{x}_{i}(t)\right) \leq-\tilde{x}_{i}(t)^{T} \rho \lambda_{2} Q \tilde{x}_{i}(t)-u_{i}(t)^{T} \rho^{2} \lambda^{2} R u_{i}(t),
$$

and integrating with respect to time along the trajectories of (15) yields

$$
V(t)-V(0) \leq-\int_{0}^{t} \tilde{x}_{i}(s)^{T}\left(\rho \lambda_{2} Q+\rho^{2} \lambda_{2}^{2} K^{T} R K\right) \tilde{x}_{i}(s) d s .
$$

The performance inequality (16) ensures the closed loop system is stable and according to Lyapunov theory $V(t) \rightarrow 0$ as $t \rightarrow \infty$ and thus $\tilde{J}_{i} \leq V\left(\tilde{x}_{i}(0)\right)$.

The inequality (16) is bilinear, and a congruence transformation $\tilde{P}=P^{-1}$ followed by a Schur complement argument yields an LMI form

$$
\underbrace{\left[\begin{array}{ccc}
\frac{1}{\rho \lambda_{2}}\left(A \tilde{P}+\tilde{P} A^{T}\right)-\left(B L+L^{T} B^{T}\right) & \tilde{P} & L^{T} \\
\tilde{P} & -Q^{-1} & \mathbf{0} \\
L & \mathbf{0} & -\frac{1}{\rho \lambda_{2}} R^{-1}
\end{array}\right]}_{\Psi(\rho)}<0,
$$

where $L=K \tilde{P}$ and $\rho \in\left\{1, \lambda_{3} / \lambda_{2}, \ldots, \lambda_{N} / \lambda_{2}\right\}$. Formally the LMI optimization problem associated with (19) is posed as:

$$
\begin{array}{cl}
\underset{Z}{\operatorname{minimize}} & \operatorname{trace}(Z) \\
\text { subject to } & {\left[\begin{array}{cc}
-Z & I \\
I & -\tilde{P}
\end{array}\right]<0,} \\
& \Psi(\rho)<0, \quad \rho \in\left\{1, \lambda_{N} / \lambda_{2}\right\} .
\end{array}
$$

Inequality (20) implies $Z>\tilde{P}^{-1}$ and so minimising $\operatorname{trace}(Z)$ minimises trace $\left(\tilde{P}^{-1}\right)=\operatorname{trace}(P)$.

\section{A. Delay analysis of single integrator network}

In the case of single integrator dynamics, for the $i^{\text {th }}$ node in (15) (for $i=2 \ldots, N$ ) the choice of $K$ which minimises the cost $J_{i}$ is

$$
\hat{K}_{i}=\frac{P_{i}}{\rho_{i} \lambda_{2} R},
$$

where $P_{i}=\sqrt{\rho_{i} \lambda_{2} Q R}$ and $\rho_{i}=\lambda_{i} / \lambda_{2}$. Since $1 \leq \rho_{i} \leq \rho_{N}$ for $i=2 \ldots N$, it follows that $\hat{K}_{i} \geq \hat{K}_{N}$ for $i=2 \ldots N$. From the gain margin properties of the LQR solution, the system $A-B K$ is stable for all $K \geq \hat{K}_{N}$ and hence the optimal solution to the minimisation problem in (20)-(21) is given by

$$
\hat{K}_{N}=\sqrt{\frac{Q}{\rho_{N} \lambda_{2} R}} .
$$

Since the node level dynamics are SISO systems, it is possible to exploit Nyquist stability arguments to compute the maximum acceptable delay before instability occurs when (15) is replaced by (14) [5]. As in [5], an appropriate loop transfer function to assess stability of the systems in (14) in the presence of delays is given by

$$
L(s)=\frac{\lambda_{N} \hat{K}_{N}}{s} e^{-\tau s} .
$$

For the system $L(s)$ in (22) the gain cross-over frequency is $\omega_{c}=\lambda_{N} \hat{K}_{N}$ and the phase at this cross over frequency is $-\left(\pi / 2+\tau \lambda_{N} \hat{K}_{N}\right)$. Hence, to ensure a positive phase-margin (and hence stability)

$$
\left(\pi / 2+\tau \lambda_{N} \hat{K}_{N}\right)<\pi \quad \Leftrightarrow \quad \tau<\frac{\pi}{2 \lambda_{N} \hat{K}_{N}} .
$$

To preserve a phase margin of $\phi_{m}$ radians, it follows that

$$
\left(\pi / 2+\tau \lambda_{N} \hat{K}_{N}\right)<\left(\pi-\phi_{m}\right) \quad \Leftrightarrow \quad \tau<\frac{\left(\pi-2 \phi_{m}\right)}{2 \lambda_{N} \hat{K}_{N}} .
$$

\section{B. Delay analysis of double integrator network}

In the double integrator case, an analytical solution to the optimization problem does not exist. Suppose the control gain obtained by solving the LMIs (20)-(21) is represented as $\hat{K}_{N}=\left[\begin{array}{ll}K_{0} & K_{1}\end{array}\right]$. As in the single integrator case, the level of delay which can be tolerated will be established using Nyquist arguments. The loop transfer function of the double integrator system in the presence of the delay is

$$
L(s)=\frac{K_{1} s+K_{0}}{s^{2}} e^{-\tau s} .
$$


The gain crossover frequency for $L(s)$ in (23) is given by

$$
\omega_{c}=\sqrt{\frac{\lambda_{N}^{2} K_{1}^{2}+\sqrt{\lambda_{N}^{4} K_{1}^{4}+4 \lambda_{N}^{2} K_{0}^{2}}}{2}},
$$

and the phase margin is

$$
\phi_{c}=\tan ^{-1}\left(\frac{\omega_{c} K_{1}}{K_{0}}\right) .
$$

Arguing as before, to ensure a positive phase margin (and hence stability in the presence of delays), we have $\tau \omega_{c}<$ $\phi_{c} \Leftrightarrow \tau<\frac{\phi_{c}}{\omega_{c}}$. To preserve a phase margin of $\phi_{m}$ radians, $\tau \omega_{c}<\phi_{c}-\phi_{m} \Leftrightarrow \tau<\frac{\phi_{c}-\phi_{m}}{\omega_{c}}$. Note that since the gains $K_{0}$ and $K_{1}$ are obtained from an LQR synthesis, it follows $\phi_{c}>\pi / 3$ [19] and so a certain level of tolerance to delays is automatically present.

\section{DESIGN APPROACH 2}

In this section, the delay $\tau$, which is assumed to be fixed and known, is explicitly accounted in the design of the gains $K$ in (3), to ensure a sub optimal level of performance with respect to the (LQR-like) cost in (4). According to Section IV, the dynamics of the multi-agents systems can be considered as a single polytopic uncertain system

$$
\begin{aligned}
\dot{x}(t) & =A x(t)-\lambda B u(t), \\
u(t) & =-K x(t-\tau),
\end{aligned}
$$

where $x \in \mathbb{R}^{n}$ and the associated cost function $J^{*}$ is given by

$$
J^{*}=\int_{0}^{\infty} x(t)^{T} \lambda Q x(t)+u(t)^{T} \lambda^{2} R u(t) d t,
$$

where $\lambda \in\left[\lambda_{2}, \lambda_{N}\right]$, and the bounds depends on the topology of the network.

Consider the Lyapunov-Krasovskii functional

$$
\begin{aligned}
V\left(x_{t}\right)= & x^{T}(t) P x(t)+\int_{t-\tau}^{t} x^{T}(s) S x(s) \mathrm{d} s \\
& +\tau \int_{t-\tau}^{t}(\tau-t+s) \dot{x}^{T}(s) Z \dot{x}(s) \mathrm{d} s .
\end{aligned}
$$

where $P \in \mathbb{S}_{n}^{+}, S \in \mathbb{S}_{n}^{+}$and $Z \in \mathbb{S}_{n}^{+}$. Differentiating the functional w.r.t. time along the trajectories of (26) yields

$$
\begin{aligned}
\dot{V}\left(x_{t}\right)= & 2 \dot{x}^{T}(t) P x(t)+x^{T}(t) S x(t)+\tau^{2} \dot{x}^{T}(t) Z \dot{x}(t) \\
& -x^{T}(t-\tau) S x(t-\tau)-\tau \int_{t-\tau}^{t} \dot{x}^{T}(s) Z \dot{x}(s) \mathrm{d} s .
\end{aligned}
$$

By applying Jensen's inequality [20] to the last integral term in (29) leads to

$$
\begin{aligned}
\dot{V}\left(x_{t}\right) & \leq 2 \dot{x}^{T}(t) P x(t)+x^{T}(t) S x(t) \\
& -x^{T}(t-\tau) S x(t-\tau)+\tau^{2} \dot{x}^{T}(t) Z \dot{x}(t) \\
& -(x(t)-x(t-\tau))^{T} Z(x(t)-x(t-\tau)) .
\end{aligned}
$$

In order to achieve a sub-optimal control design, the cost in (27) is manipulated by a modified functional

$$
W\left(x_{t}\right)=\dot{V}\left(x_{t}\right)+\lambda x^{T}(t) Q x(t)+u^{T}(t) \lambda^{2} R u(t),
$$

where the matrices $Q$ and $R$ are symmetric positive definite matrices associated with the cost (27).

Note that, for any matrix $Y \in \mathbb{R}^{3 n \times n}$, the equality

$$
\xi^{T}(t) Y(A x(t)+B \lambda u(t)-\dot{x}(t))=0
$$

holds, where $\xi(t)^{T}:=\left[\begin{array}{lll}x(t)^{T} & x(t-\tau)^{T} & \dot{x}(t)^{T}\end{array}\right]$. Since $u(t)=-K x(t-\tau)$, the right hand side of (31) can be written in terms of $\xi(t)$ as

$$
\begin{aligned}
W\left(x_{t}\right) \leq & \xi^{T}(t)\left[\begin{array}{ccc}
S-Z+Q & Z & P \\
Z & -S-Z & 0 \\
P & 0 & \tau^{2} Z
\end{array}\right] \xi(t) \\
& +\xi^{T}(t)\left[\begin{array}{c}
0 \\
\lambda K^{T} \\
0
\end{array}\right] R\left[\begin{array}{c}
0 \\
\lambda K^{T} \\
0
\end{array}\right]^{T} \xi(t) \\
& +2 \xi^{T}(t) Y\left[\begin{array}{lll}
A & -\lambda B K & -I
\end{array}\right] \xi(t) .
\end{aligned}
$$

Assume that the matrix $Y$ has the following structure $Y^{T}=\left[\begin{array}{lll}\left.\overline{(} Y^{-1}\right)^{T} & 0 & \bar{\epsilon}\left(Y^{-1}\right)^{T}\end{array}\right]$, where $\epsilon$ is a positive scalar and the matrix $\bar{Y}$ in $\mathbb{R}^{n \times n}$ is nonsingular. This manipulation corresponds to the use of the descriptor approach proposed in [21]. Define $\bar{\xi}(t)^{T}=$ $\left[\begin{array}{lll}\left(\bar{Y}^{-1} x(t)\right)^{T} & \left(\bar{Y}^{-1} x(t-\tau)\right)^{T} & \left(\bar{Y}^{-1} \dot{x}(t)\right)^{T}\end{array}\right] . \quad$ Then $W(\cdot)$ can be rewritten as

$$
\begin{aligned}
& W\left(x_{t}\right) \leq \bar{\xi}^{T}(t)\left(\left[\begin{array}{ccc}
\bar{S}-Z & \bar{Z} & \bar{P} \\
\bar{Z} & -\bar{S}-\bar{Z} & 0 \\
\bar{P} & 0 & \tau^{2} \bar{Z}
\end{array}\right]\right. \\
& +2\left[\begin{array}{c}
I \\
0 \\
\epsilon I
\end{array}\right]\left[\begin{array}{c}
\bar{Y}^{T} A^{T} \\
-\lambda \bar{Y}^{T} K^{T} B^{T} \\
-\bar{Y}^{T}
\end{array}\right]^{T} \\
& +\left[\begin{array}{c}
0 \\
\lambda \bar{Y}^{T} K^{T} R \\
0
\end{array}\right] R^{-1}\left[\begin{array}{c}
\overline{0} \\
\lambda \bar{Y}^{T} K^{T} R \\
0
\end{array}\right]^{T} \\
& \left.+\left[\begin{array}{c}
\bar{Y}^{T} Q \\
0 \\
0
\end{array}\right] Q^{-1}\left[\begin{array}{c}
\bar{Y}^{T} Q \\
0 \\
0
\end{array}\right]^{T}\right) \bar{\xi}(t),
\end{aligned}
$$

where $\bar{P}=\bar{Y}^{T} P \bar{Y}, \bar{S}=\bar{Y}^{T} S \bar{Y}, \bar{Z}=\bar{Y}^{T} Z \bar{Y}$. Finally, defining the matrix variable $\bar{K}=K \bar{Y}$ and applying the Schur complement ensures that the functional (33) is negative definite provided the LMI condition

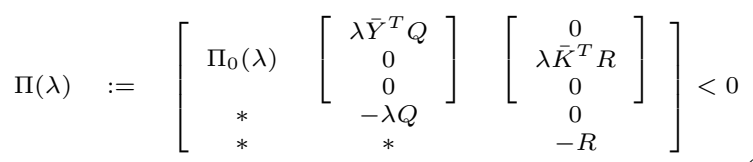

for all values of $\lambda \in\left[\lambda_{\min }, \lambda_{\max }\right]$ where

$$
\begin{aligned}
\Pi_{0}(\lambda) & =\left[\begin{array}{ccc}
\bar{S}-\bar{Z} & \bar{Z} & \bar{P} \\
\bar{Z} & -\bar{S}-\bar{Z} & 0 \\
\bar{P} & 0 & \tau^{2} \bar{Z}
\end{array}\right] \\
& +\mathcal{H} e\left\{\left[\begin{array}{c}
I \\
0 \\
\epsilon I
\end{array}\right]\left[\begin{array}{c}
\bar{Y}^{T} A^{T} \\
-\lambda \bar{K}^{T} B^{T} \\
-\bar{Y}^{T}
\end{array}\right]\right\}
\end{aligned}
$$

Since the LMI is affine in the parameter $\lambda$, the condition $\Pi(\lambda)<0$ is equivalent to solving the two LMIs $\Pi\left(\lambda_{\text {min }}\right)<$ 0 and $\Pi\left(\lambda_{\max }\right)<0$. Then if these two conditions hold, the functional $W(\cdot)$ is negative definite. Integrating $W(\cdot)$ in (33) over the interval $[0, T]$ ensures that

$V\left(x_{T}\right)-V\left(x_{0}\right)+\int_{0}^{T}\left(x^{T}(t) \lambda Q x(t)+u^{T}(t) \lambda^{2} R u(t)\right) d t<0$.

Since $V\left(x_{T}\right)>0$, a bound of the performance index is given by letting $T$ tend to infinity

$$
\int_{0}^{\infty}\left(x^{T}(t) \lambda Q x(t)+u^{T}(t) \lambda^{2} R u(t)\right) d t<V\left(x_{0}\right)
$$


From the definition of the Lyapunov-Krasovskii functional,

$$
\begin{aligned}
V\left(x_{0}\right) & =x^{T}(0) \bar{Y}^{-T} \bar{P}_{\bar{Y}}^{-1} x(0) \\
& +\int_{-\tau}^{0} x^{T}(s) \bar{Y}^{-T} \bar{S}^{-1} x(s) \mathrm{d} s \\
& +\int_{-\tau}^{0}(\tau+s) \dot{x}^{T}(s) \bar{Y}^{-T} \bar{Z} \bar{Y}^{-1} \dot{x}(s) \mathrm{d} s .
\end{aligned}
$$

From (36) and (37)

$$
J^{*} \leq \mu_{\bar{P}}|x(0)|^{2}+\tau \mu_{\bar{S}}\left\|x_{0}\right\|_{\tau}^{2}+\mu_{\bar{Z}} \tau^{2} / 2\left\|\dot{x}_{0}\right\|_{\tau}^{2},
$$

where, for $M=\bar{P}, \bar{S}, \bar{Z}, \mu_{M}$ is the largest eigenvalue $\bar{Y}^{-1} \bar{M} \bar{Y}$.

Optimization: The optimization corresponds to the minimization of $\mu_{\bar{P}}, \mu_{\bar{S}}$ and $\mu_{\bar{Z}}$. Consider first the matrix $\bar{P}$. Introduce the parameter $\mu$ such that $\mu_{\bar{P}} \leq \mu$ which can be re-written in the form of a matrix inequality as follows:

$$
\bar{Y}^{-T} \bar{P} \bar{Y}^{-1} \leq \mu I .
$$

Applying the Schur complement yields

$$
\left[\begin{array}{cc}
\mu I & I \\
I & \bar{Y}^{T} \bar{P}^{-1} \bar{Y}
\end{array}\right]>0 .
$$

Since $\left(\bar{Y}^{T}-\bar{P}\right) \bar{P}^{-1}(\bar{Y}-\bar{P}) \geq 0$, it follows that

$$
\bar{Y}^{T} \bar{P}^{-1} \bar{Y} \geq \bar{Y}+\bar{Y}^{T}-\bar{P}
$$

This means that if condition (40) holds, then (39) holds.

Formally the LMI optimisation problem associated with (34) is posed as:

$$
\begin{aligned}
\underset{\bar{P}, \bar{S}, \bar{Z}, \bar{Y}, \epsilon}{\operatorname{minimize}} & \mu \\
\text { subject to } & \Pi\left(\lambda_{\min }\right)<0, \\
& \Pi\left(\lambda_{\max }\right)<0, \\
& \Psi(\bar{P})>0, \\
& \Psi(\bar{S})>0, \\
& \Psi(\bar{Z})>0 .
\end{aligned}
$$

where $\bar{P}, \bar{S}$ and $\bar{Z}$ in $\mathbb{S}_{n}^{+}$and

$$
\Psi(M)=\left[\begin{array}{cc}
\mu I & I \\
I & \bar{Y}+\bar{Y}^{T}-M
\end{array}\right], \quad \forall M \in \mathbb{S}_{n}^{+} .
$$

The solution of this convex optimisation problem is obtained using the standard mincx solver in the MATLAB LMI toolbox. The gain matrix $K$ is computed by $K=\bar{K} \bar{Y}^{-1}$, and the associated control law

$$
u_{i}(t)=-K z_{i}(t),
$$

guarantees that the closed loop system (7) is asymptotically stable for the known fixed delay $\tau>0$. Moreover the cost functions (4) satisfy

$$
J_{i} \leq \mu\left(|x(0)|^{2}+\tau\left\|x_{0}\right\|_{\tau}^{2}+\tau^{2} / 2\left\|\dot{x}_{0}\right\|_{\tau}^{2}\right) .
$$

\section{Results}

The computations have been done in the MATLAB/SIMULINK environment. All the simulations are carried out using an ODE1 (Euler) integration scheme with a step size fixed at 0.001 .
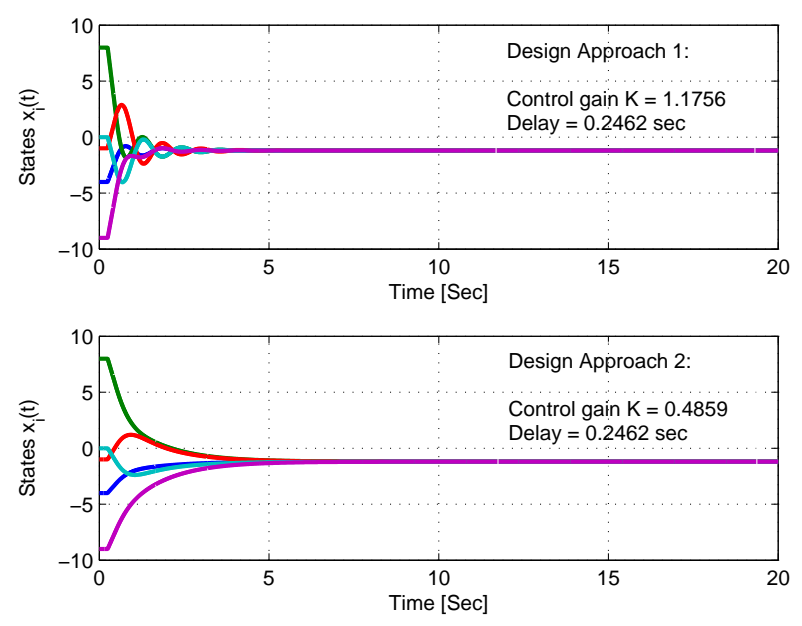

Fig. 1: Time evolution of states, showing average consensus

\section{A. Single integrator type consensus laws}

For single integrator type consensus laws, the control law (3) is designed to minimise the cost defined in (4), where the constants associated with the performance costs are chosen as $Q=5$ and $R=1$. A nearest neighbour topology is assumed for the network with five identical nodes. Following the design Approach 1 (assuming $\tau=0$ ), the consensus control gain in (3) is determined as $K=\sqrt{\frac{Q}{\lambda_{N} R}}=1.1756$, where $\lambda_{N}=3.618$ is the maximum eigenvalue of the Laplacian matrix corresponding to the network of five nodes with nearest neighbour coupling. An identical solution can be determined by solving the formal polytopic LMI optimization problem in (20)-(21). Furthermore, from the delay margin analysis in section IV-A, a positive phase margin, i.e., stability, is ensured for all delays $\tau<0.3693$.

Consider two cases associated with required phase margins of $\phi_{m}=30^{\circ}$ and $\phi_{m}=60^{\circ}$. Following the analysis in section IV-A, to preserve the phase margins $\phi_{m}=30^{\circ}$ and $\phi_{m}=60^{\circ}$, the acceptable delay bounds are $\tau_{\phi_{m}=30^{\circ}}=$ 0.2462 and $\tau_{\phi_{m}=60^{\circ}}=0.1231$ respectively. For each of these desired delay limits (delay $\tau$ is fixed and known), the design Approach 2 is followed and the optimisation problem (41)(45) is solved (with the same $Q$ and $R$ values) to obtain the consensus control gain $K=0.4859$ (corresponds to $30^{\circ}$ phase margin) and $K=0.5170$ (corresponds to $60^{\circ}$ phase margin) respectively.

In figure 1, the time evolution of the dynamics of the network of 5 agents defined in (1) for the case of $30^{\circ}$ phase margin is shown. The time responses depicted in the upper subplot of figure 1 is associated with the consensus control gain $K=1.1756$ obtained by the classical LQR-like design Approach 1, but in the presence of a delay $\tau=0.2462$. The time responses in the lower subplot of figure 1 correspond to the consensus control gain $K=0.4859$ synthesized by design Approach 2; an identical delay $\tau=0.2462$ is present, but the time responses are significantly better (less oscillatory) to that from the initial design. 

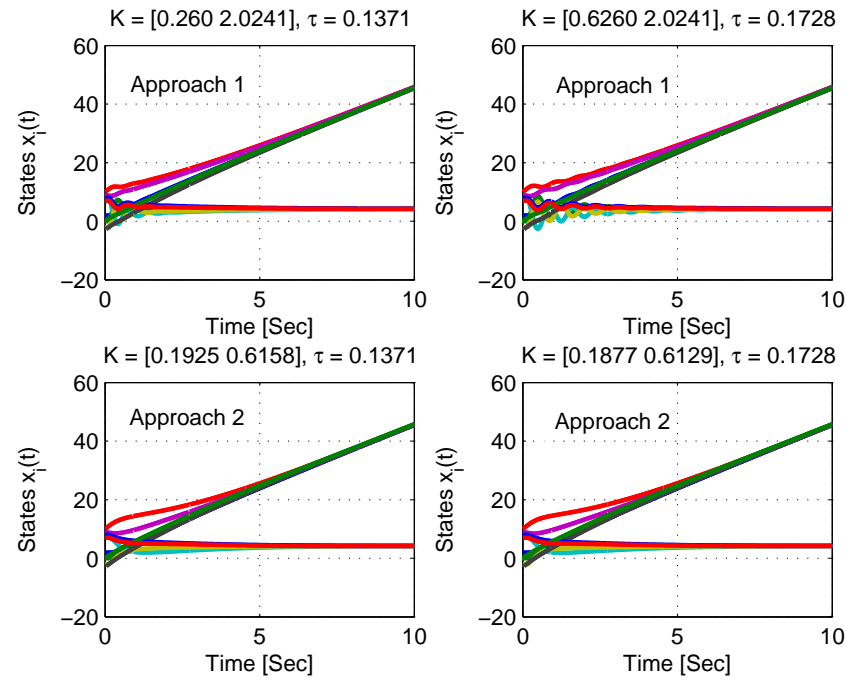

Fig. 2: Comparison of state history achieving average consensus with different designs in double integrator case

\section{B. Double integrator type consensus laws}

For the double integrator case, the consensus control law (3) is designed to minimise the cost defined in (4), where the constants associated with the performance cost are chosen as $Q=\left[\begin{array}{lll}1 & 0 ; 0 & 10\end{array}\right]$ and $R=1$. An identical network as in the single integrator case is considered. As in the single integrator case (scalar design problem), it is not trivial to find the gain using an analytical expression. Following, the LMI optimization problem in (20) - (21), the consensus control gain in (3) is determined as $K=\left[\begin{array}{ll}0.6260 & 2.0241\end{array}\right]$. Following the delay margin analysis in section IV-B, a positive phase margin, i.e., stability, is ensured for all delays $\tau<0.2086$.

Two cases of phase margins $\phi_{m}=30^{\circ}$ and $\phi_{m}=15^{\circ}$ are considered. Following the analysis in section IV-A, to preserve the phase margins $\phi_{m}=30^{\circ}$ and $\phi_{m}=15^{\circ}$, the acceptable delay bounds are $\tau_{\phi_{m}=30^{\circ}}=0.1371$ and $\tau_{\phi_{m}=15^{\circ}}=0.1728$ respectively. For each of these desired fixed delay limits, design approach 2 is followed (with identical $Q$ and $R$ matrices as used in design 1); the optimisation problem in (41)-(45) is solved to obtain the consensus control gain $K=\left[\begin{array}{ll}0.1925 & 0.6158\end{array}\right]$ (corresponding to $30^{\circ}$ phase margin) and $K=[0.18770 .6129]$ (corresponding to $15^{\circ}$ phase margin) respectively. The time evolution of the dynamics of the network of 5 agents defined in (1) for the different cases (see the titles of each subplot) is depicted in figure 2 .

\section{CONCLUSIONS}

In this paper two different approaches are proposed for developing consensus control laws based on homogenous self delayed relative information. One approach is rooted in a classical LQR approach and the second one is based on a novel linear matrix inequality condition developed explicitly to address the LQR like performance problem in the presence of fixed, known delay. Both have been compared by using single and double integrator type consensus formulations.

\section{REFERENCES}

[1] Olfati-Saber, R. and Murray, R.M. (2004), "Consensus Problems in Networks of Agents with Switching Topology and Time-Delays," IEEE Transactions on Automatic Control, 49, 1520-1533.

[2] Bliman, P.-A., and Ferrari-Trecate, G. (2008)"Average consensus problems in networks of agents with delayed communications," Automatica, 44, 1985-1995.

[3] Seuret, A., Dimarogonas, D.V., and Johanson, K.H. (2008), "Consensus under Communication Delays," Proceedings of IEEE Conference on Decision and Control, Cancun, Mexico.

[4] Münz, U., Papachristodoulou, A., and Allgöwer, F. (2008), "DelayDependent Rendezvous and Flocking of Large Scale Multi-Agent Systems with Communication Delays," Proceedings of IEEE Conference on Decision and Control, Cancun, Mexico.

[5] Münz, U., Papachristodoulou, A., and Allgöwer, F. (2010), "Delay robustness in consensus problems," Automatica, 46, 1252-1265.

[6] Papachristodoulou, A., Jadbabaie, A., and Münz, U. (2010) "Effects of Delay in Multi-Agent Consensus and Oscillator Synchronization," IEEE Transactions on Automatic Control, 55, 1471-1477.

[7] Yang, W., Bertozzi, A.L., and Wang, X.F. (2008), "Stability of a second order consensus algorithm with time delay," Proceedings of IEEE Conference on Decision and Control, Cancun, Mexico.

[8] Cepeda-Gomez, R. and Olgac, N. (2011), "Consensus analysis with large and multiple communication delays using spectral delay space concept," International Journal of Control, 84, 1996-2007.

[9] Qin, J., Gao, H., and Zheng, W.X. (2011), "Second order consensus for multi-agent systems with switching topology and communication delay," Systems and Control Letters, 60, 390-397.

[10] Deshpande, P., Menon, P., P., and Edwards, C. (2013), "Delayed static output feedback control of a network of double integrators," Automatica, 49, 3498-3501.

[11] Wen, G., Duan, Z., Yu, W., and Chen, G. (2012), "Consensus of multiagent systems with nonlinear dynamics and sampled-data information: a delayed-input approach," International Journal of Robust and Nonlinear Control, 23, 602-619.

[12] Liu, Y. and Jia, Y. (2011), " $\mathcal{H}_{\infty}$ consensus control for multi-agent systems with linear coupling dynamics and communication delays,' International Journal of System Science, 43, 50-62.

[13] Hirche, S., Matiakis, T., and Buss, M. (2009), "A Distributed Controller Approach for Delay-Independent Stability of Networked Control Systems," Automatica, 45, 828-1836.

[14] Tian, E., Yue, D., and Gu, Z. (2010) "Robust $\mathcal{H}_{\infty}$ control for nonlinear system over a network: A piecewise analysis method," Fuzzy Sets and Systems, 161, 2731-2745.

[15] Godsil, C., and Royle, G. (2001) "Algebraic Graph Theory," Springer.

[16] Mesbhahi, M., and Egerstedt, M. (2010), "Graph theoretic methods in multiagents systems," Princeton series in applied mathematics, Princeton, 2010.

[17] Wu, C. W. (2007), "Synchronisation of complex networks of nonlinear dynamical systems," World scientific publishing company.

[18] Gahinet, P., Nemirovski, A., Laub, A., and Chilali, M. (1995) LMI Control Toolbox, User Guide. MathWorks, Inc.

[19] Maciejowski, J. M., (1989), “Multivariable feedback design,” AddisonWesley Publishing Company.

[20] Gu, K., Kharitonov, V.-L., and Chen, J. (2003) "Stability of time-delay systems," Birkhauser.

[21] Fridman, E. and Shaked, U. (2002), "An improved stabilization method for linear time-delay systems" IEEE Transactions on Automatic Control Vol. 47(11), 1931-1937. 